\title{
Life on the Edge: Characterising the Edges of Mutually Non-dominating Sets
}

\author{
Richard M. Everson \\ R.M.Everson@exeter.ac.uk \\ Department of Computer Science, University of Exeter, Exeter, UK \\ David J. Walker \\ D.J.Walker@exeter.ac.uk \\ Department of Computer Science, University of Exeter, Exeter, UK \\ Jonathan E. Fieldsend \\ J.E.Fieldsend@exeter.ac.uk \\ Department of Computer Science, University of Exeter, Exeter, UK
}

doi:10.1162/EVCO_a_00124

\begin{abstract}
Multi-objective optimisation yields an estimated Pareto front of mutually nondominating solutions, but with more than three objectives, understanding the relationships between solutions is challenging. Natural solutions to use as landmarks are those lying near to the edges of the mutually non-dominating set. We propose four definitions of edge points for many-objective mutually non-dominating sets and examine the relations between them. The first defines edge points to be those that extend the range of the attainment surface. This is shown to be equivalent to finding points which are not dominated on projection onto subsets of the objectives. If the objectives are to be minimised, a further definition considers points which are not dominated under maximisation when projected onto objective subsets. A final definition looks for edges via alternative projections of the set. We examine the relations between these definitions and their efficacy in many dimensions for synthetic concave- and convex-shaped sets, and on solutions to a prototypical many-objective optimisation problem, showing how they can reveal information about the structure of the estimated Pareto front. We show that the "controlling dominance area of solutions" modification of the dominance relation can be effectively used to locate edges and interior points of high-dimensional mutually non-dominating sets.
\end{abstract}

\section{Keywords}

Many-objective optimisation, dominance, edges, preference ordering, visualisation.

\section{Introduction}

As optimisation algorithms become capable of tackling multi-objective problems with at least four objectives, often called many-objective problems, it becomes important to find ways of understanding and visualising the solutions in the approximation to the many-objective Pareto front (Ishibuchi et al., 2008). Recently a number of methods have been developed to map high-dimensional sets of mutually non-dominating of solutions to the plane or three dimensions for visualisation (e.g., Obayashi, 2002; Yoshikawa et al., 2007; Tušar and Filipič, 2011; Kudo and Yoshikawa, 2012; Walker et al., 2013; Fieldsend and Everson, 2013). However, the inevitable loss of information concomitant with the dimension reduction means that the problem owner or decision maker may need help in navigating the visualisation. One way of doing that is to identify landmark 


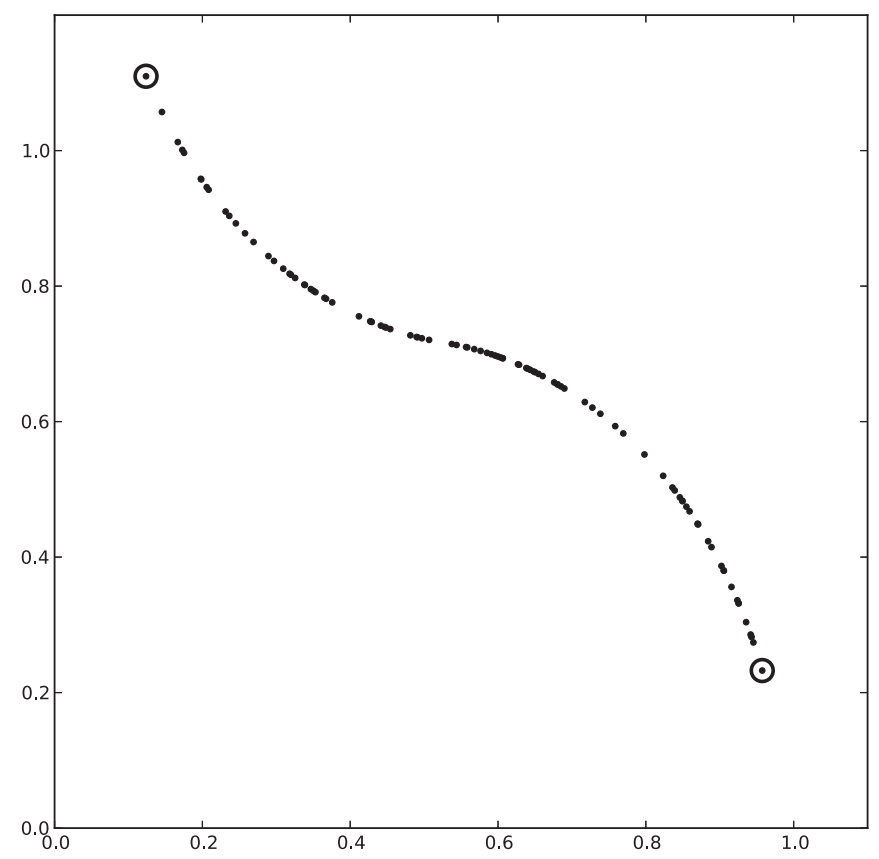

Figure 1: A two objective mutually non-dominating set with the two extremal individuals, corresponding to the edges, marked with circles.

solutions-individuals with known properties-against which other individuals may be compared. Natural landmark individuals are extremal in one sense or another. For example, the individuals that maximise or minimise any single objective provide natural reference points (Walker et al., 2013). Singh et al. (2011) have given a procedure for finding the corners in multi-objective optimisation problems which may be thought of as the locations where more than one objective is extremised. In this paper we extend these ideas by examining what is meant by the edge of a mutually non-dominating set.

Although we concentrate on the visualisation aspects of edge points, they are also of interest for the design of multi-objective evolutionary algorithms, where it is useful to preferentially retain edge points in a search population, because these points preserve the spread of the search and are inherently diverse (e.g., Knowles and Corne, 2000; Zitzler et al., 2002; Smith et al., 2008).

With two objectives the idea of edges is intuitively straightforward: as illustrated in Figure 1, the two individuals lying at the ends of the set comprise the edges and all the other individuals lie in the interior. We assume throughout that the criteria are to be minimised. Likewise, with three objectives the observer of a scatter plot of a mutually non-dominating set such as that shown in Figure 2 can readily identify which points are close to the edges of the set and which points are in the interior. Nonetheless, as we discuss later, defining precisely which individuals comprise the edges in this case is not entirely straightforward. Identifying these edges is important for understanding the extent of the set, although it is generally unlikely that a decision maker, in choosing one particular solution from the set, will pick one of the edge individuals, rather preferring another solution that makes a trade-off between all the objectives. With more than three objectives the edge points cannot be directly identified visually and it is not a priori 

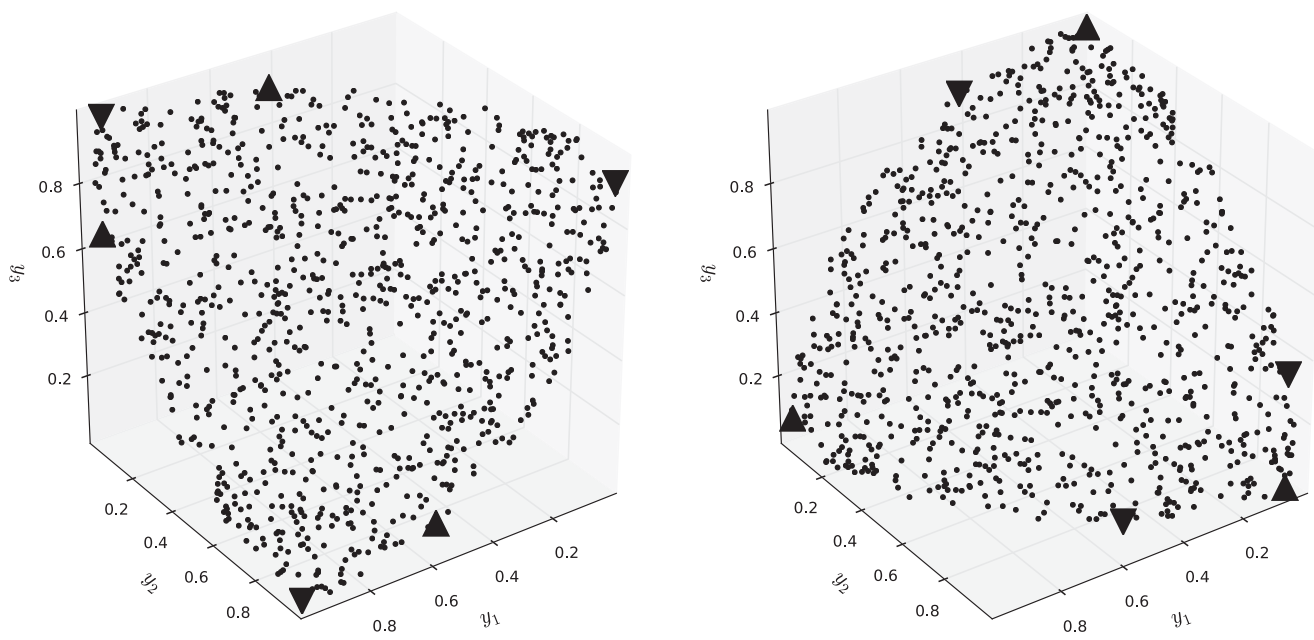

Figure 2: Three objective concave and convex mutually non-dominating sets showing extremal individuals. Individuals minimising and maximising objectives are marked with $\boldsymbol{\nabla}$ and $\boldsymbol{\Delta}$ respectively. The convex set comprises 1000 points, $\mathbf{y}$, uniformly sampled from the positive octant a shell of unit radius. The concave set is generated from the positive set by $\mathbf{y} \mapsto-\mathbf{y}+(1,1,1)^{T}$.

clear that visualisation methods that map the set to the plane also map the edges in the original high-dimensional objective space to the edges of the planar visualisation.

In the body of this paper we examine three definitions of the edges of a mutually non-dominating set, all of which generalise the basic two-objective notion. Briefly, the first of these defines edge points as those that extend the range of the attainment surface. This turns out to identify the same edge individuals as a generalisation of the "corner points" defined by Singh et al. (2011) and is closely related to the efficiency of individuals after projection onto subsets of the objectives and preference ordering (Das and Dennis, 1998; di Pierro et al., 2007). The third definition generalises these ideas to consider points that are non-dominated under maximisation rather than minimisation, which leads to consideration of the attainment surface under maximisation. This third definition most closely corresponds to most people's intuitive idea of where the edge points lie. In the Appendix, we give an alternative definition from another perspective in which the mutually non-dominating set is projected onto an alternative lower-dimensional subspace before edges are located. Some of the material here was presented in Everson et al. (2013), and in this paper we formalise the definitions and theorems, provide additional demonstrations of the methods using synthetic data specifically designed to highlight features of many-objective non-dominated sets using edges, and investigate the utility of cone-based dominance indicators for identifying edges.

We are particularly concerned with the performance of these definitions for manyobjective sets. With many criteria and relatively small sets, almost all points become edge points. In addition to synthetic two- and three-dimensional datasets, which are useful because the edge points are easily visualised, we therefore examine these methods on two 9-objective datasets. One is for a problem proposed as a prototypical manyobjective problem by Hughes (2007a, 2007b), while the other is a synthetic dataset created from common geometrical features found in non-dominated sets. We show that 


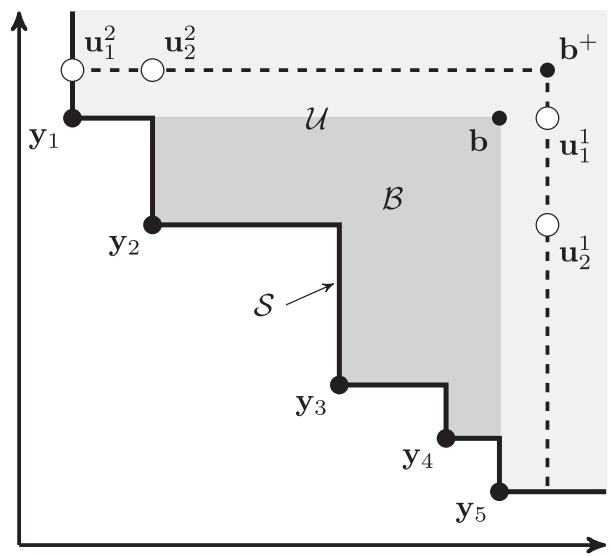

Figure 3: A mutually non-dominating set of points $\mathcal{Y}=\left\{\mathbf{y}_{i}\right\}$ and the region that it dominates, $\mathcal{U}$. The attainment surface $\mathcal{S}$ is shown with the thick black line. Points in the region $\mathcal{B}$ dominate the reference point $\mathbf{b}$, whose $m$ th coordinate is $b_{m}=\max _{\mathbf{y}^{\prime} \in \mathcal{Y}} y_{m}^{\prime}$. Candidate points for sole domination by $\mathbf{y}_{1}\left(\mathbf{u}_{1}^{1}\right.$ and $\left.\mathbf{u}_{1}^{2}\right)$ and $\mathbf{y}_{2}\left(\mathbf{u}_{2}^{1}\right.$ and $\left.\mathbf{u}_{2}^{2}\right)$ are denoted by white circles; see Equation (6). In fact, $\mathbf{u}_{1}^{2}$ is only dominated by $\mathbf{y}_{1}$, so $\mathbf{y}_{1}$ is an edge point. None of the candidates for sole domination of $\mathbf{y}_{2}$ are dominated exclusively by $\mathbf{y}_{2}$; hence $\mathbf{y}_{2}$ is not an edge point.

edge points that are close to being efficient in the preference ordering sense tend to lie close to the edges of low-dimensional visualisations of the set and we examine the use of the controlling dominance area of solutions (CDAS; Sato et al., 2007a) method for identifying strong edges in many criterion sets, which further extends the work presented in Everson et al. (2013).

To be definite, we assume that the set of mutually non-dominating individuals $\mathcal{Y}=\left\{\mathbf{y}_{n}\right\}_{n=1}^{N}$ comprises $N$ individuals $\mathbf{y}_{n}$, each of which is an $M$-dimensional vector of objective values; $y_{n m}$ denotes the value of the $m$ th objective for the $n$th point. Without loss of generality, we assume that minimising the objective values corresponds to good performance.

\section{Attainment Surface Edges}

The idea underlying this characterisation of the edge points of $\mathcal{Y}$ is that they extend the attainment surface (Zitzler, 1999); that is, they dominate (unbounded) regions that are not dominated by other elements of $\mathcal{Y}$. This is illustrated for a two-dimensional set in Figure 3, in which the region $\left\{\left(u_{1}, u_{2}\right) \mid y_{11}<u_{1} \leq y_{21}\right.$ and $\left.y_{12}<u_{2}\right\}$ is dominated only by $\mathbf{y}_{1}$. This region is unbounded, but note that there are regions close to the attainment surface which are also dominated by a single element of $\mathcal{Y}$, but are bounded. For example, only $\mathbf{y}_{3}$ dominates the rectangle $\left\{\left(u_{1}, u_{2}\right) \mid y_{31}<u_{1}<y_{41}\right.$ and $\left.y_{32}<u_{2}<y_{22}\right\}$. Our definition of an edge point of $\mathcal{Y}$ is thus a point which extends the region dominated by the attainment surface by appending an unbounded region.

To make this precise, define the region dominated by $\mathcal{Y}$ :

$$
\mathcal{U}=\{\mathbf{u} \mid \mathbf{y} \prec \mathbf{u} \text { for some } \mathbf{y} \in \mathcal{Y}\}
$$


The attainment surface is the boundary of $\mathcal{U}: \mathcal{S}=\partial \mathcal{U}$ (Smith et al., 2008). Also, let $\mathbf{b}$ be the point which has the maximum coordinate of any element of $\mathcal{Y}$ in each dimension:

$$
b_{m}=\max _{\mathbf{y}_{n} \in \mathcal{Y}} y_{n m} .
$$

Then let $\mathcal{B}$ be the region which is dominated by $\mathcal{Y}$ but lies "below" $\mathbf{b}$ :

$$
\mathcal{B}=\mathcal{U} \cap\{\mathbf{y} \mid \mathbf{y} \prec \mathbf{b}\} .
$$

Finally, we define a function which returns the set of the elements of $\mathcal{Y}$ that (weakly) dominate a point $\mathbf{y}$ :

$$
\operatorname{doms} \mathcal{Y}(\mathbf{y})=\{\mathbf{u} \in \mathcal{Y} \mid \mathbf{u} \preceq \mathbf{y}\} .
$$

Given these preliminaries, we say that $\mathbf{y} \in \mathcal{Y}$ is an attainment surface edge point if there are points outside $\mathcal{B}$ (i.e., sufficiently far away from $\mathcal{Y}$ ) that are dominated by $\mathbf{y}$ alone.

Definition 1 (AtTAinment SuRface Edge Point): A point $\boldsymbol{y} \in \mathcal{Y}$, a mutually non-dominating set, is an attainment surface edge point of $\mathcal{Y}$ if and only if there exists a $\boldsymbol{u} \in \mathcal{U} \backslash \mathcal{B}$ such that $\operatorname{doms}_{\mathcal{Y}}(\boldsymbol{u})=\{\boldsymbol{y}\}$.

In order to determine whether an element $\mathbf{y} \in \mathcal{Y}$ is the sole dominator of some point $\mathbf{u} \in \mathcal{U} \backslash \mathcal{B}$ we observe (see Figure 3) that points which might be dominated by a single $\mathbf{y}_{n}$ lie "directly above" or "directly to the side" of $\mathbf{y}_{n}$. With this in mind, candidates for sole domination can be constructed by extending each $\mathbf{y}_{n}$ parallel to each objective axis in turn into $\mathcal{U} \backslash \mathcal{B}$ and testing how many elements of $\mathcal{Y}$ dominate it. We choose a particular hyper-rectangle to project onto, defined in terms of a point $\mathbf{b}^{+} \in \mathcal{U} \backslash \mathcal{B}$, whose coordinates are:

$$
b_{m}^{+}=b_{m}+\epsilon, \quad \epsilon>0, \quad m=1, \ldots, M .
$$

So long as it is positive, the value of $\epsilon$ is immaterial. If the dimension of $\mathbf{y}$ is $M$, then, as illustrated in Figure 3, $M$ candidate points $\mathbf{u}_{n}^{m}, m=1, \ldots, M$ corresponding to $\mathbf{y}_{n}$ are constructed with coordinates:

$$
u_{n i}^{m}=\left\{\begin{array}{ll}
y_{n i} & i \neq m \\
b_{m}^{+} & i=m
\end{array} \quad i=1, \ldots, M .\right.
$$

By construction $\mathbf{y}_{n} \prec \mathbf{u}_{n}^{m}$ for all $m$, but if each of the $\mathbf{u}_{n}^{m}$ is dominated by at least one other element of $\mathcal{Y}$ (besides $\mathbf{y}_{n}$ ) then $\mathbf{y}_{n}$ is not an edge point. In Figure $3, \mathbf{u}_{2}^{1}$ is dominated by $\mathbf{y}_{3}, \mathbf{y}_{4}$, and $\mathbf{y}_{5}$, and $\mathbf{u}_{2}^{2}$ is dominated by $\mathbf{y}_{1}$. However, $\mathbf{u}_{1}^{2}$ is dominated only by $\mathbf{y}_{1}$, which is therefore an edge point.

Figure 4 presents three examples of edge identification using the attainment surface. Figure 4(a) shows the edge points identified in the concave set shown in Figure 2. Here the method has identified points that agree reasonably with intuition. However, on the convex set, Figure 4(b) shows that the method fails to identify many of the points that might be expected to lie in the edge set. The reason for this lies with points such as the two edge points labelled $\alpha$ and $\beta$. In terms of the first two objective coordinates $y_{1}$ and $y_{2}, \alpha$ and $\beta$ are near-optimal. It is therefore unlikely that another element of the set will dominate regions with respect to these coordinates that are not also dominated by $\alpha$ and $\beta$. Consequently, few of the individuals in the convex population are on the edge; the relatively few individuals that are identified as edge points either have $y_{1}$ or $y_{2}$ coordinates smaller than those of $\alpha$ or $\beta$, or have close to optimal $y_{3}$ coordinates. In a similar manner, points lying close to the $y_{1}$ and $y_{3}$ axes determine the extent of the attainment surface in those directions. Figure $4(\mathrm{c})$ shows the edge points of 


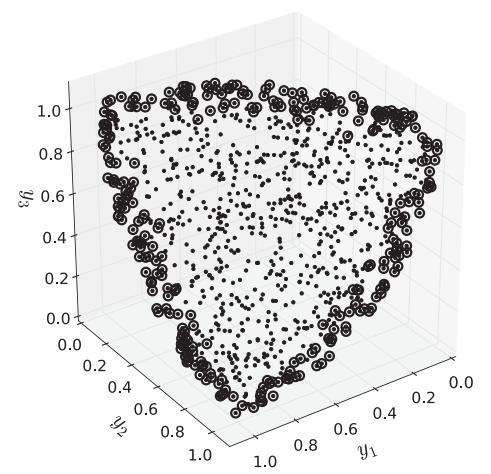

(a) Concave

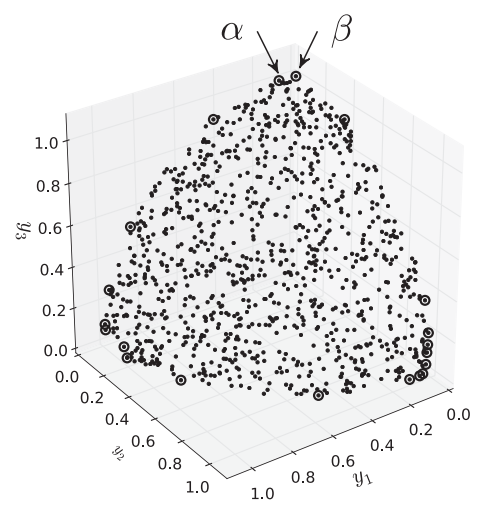

(b) Convex

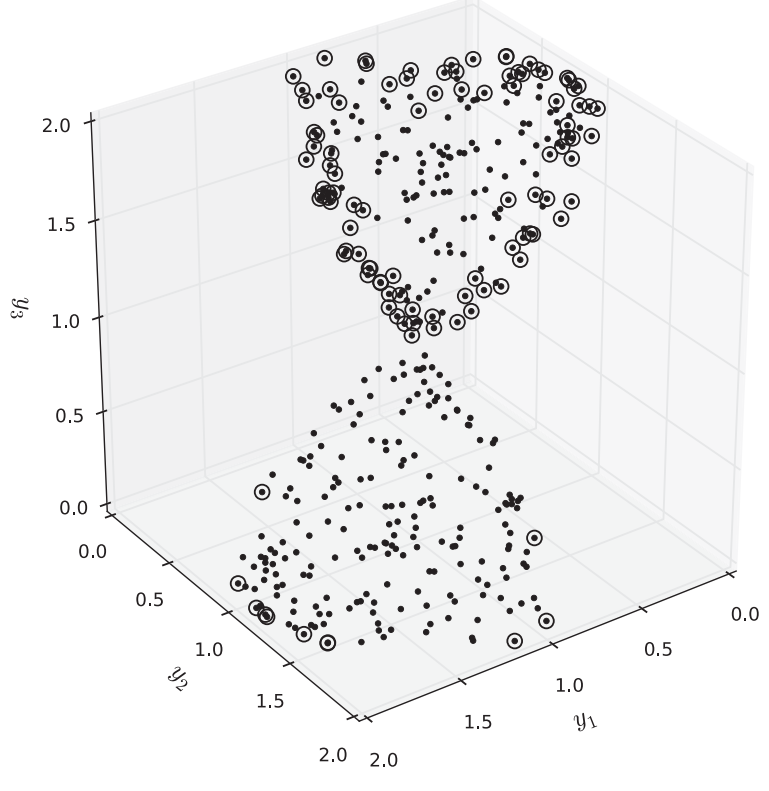

(c) Hourglass

Figure 4: Edge points (circles) identified by the attainment surface method for (a) concave, (b) convex, and (c) hourglass sets.

an hourglass set, which was constructed from the union of samples of appropriately translated concave and convex sets and thus incorporates concave and convex regions. Here too, the edge points of the concave region have been well identified, but points that are intuitively close to the edge of the convex region do not correspond to this definition.

Direct application of this definition to high dimensional sets is not successful because almost all elements of the set are identified as edge points. We find that all but two of the 11,000 individuals in a mutually non-dominating 9-dimensional criterion set (Hughes, 2007a, 2007b) are edge points, with similar outcomes for synthetic sets in many dimensions. This is to be expected, as it is a side effect of the inability of the dominance relation to discriminate between individuals in high-dimensional spaces (Farina and Amato, 2003).

\section{From Corners to Edges}

We recall the definition of corner points given by Singh et al. (2011). At first glance, these appear to be good candidates for extremal points lying on the edge of a mutually 
non-dominating set. They consider the minimisation of $M$ functions $f_{i}(\mathbf{x})$ where $\mathbf{x}$ is a vector of decision variables. If, when minimising a subset of $k<M$ objectives, there exists a single minimising point, then that point is a corner point. Clearly, in non-degenerate cases, there are at least $M$ corner points, each corresponding to the minimisation of a single objective, but there are $2^{M}-1$ possible combinations to be tested.

The definition of corner points might be adapted to a (finite) set of mutually nondominating points as follows. Let $\kappa$ denote a set of indices and let $\mathbf{y}_{n}^{\kappa}$ be the projection of $\mathbf{y}_{n}$ onto the indices indicated by $\kappa$. If $|\kappa|=k$, then $\mathbf{y}_{n}^{\kappa}$ is a $k$-dimensional vector. Also, let the function nondom $(\mathcal{A})$ be the function that returns the maximal set of non-dominated members of the set $\mathcal{A}$ :

$$
\operatorname{nondom}(\mathcal{A})=\{\mathbf{u} \in \mathcal{A} \mid \nexists(\mathbf{v} \in \mathcal{A} \wedge \mathbf{v} \prec \mathbf{u})\}
$$

Then $\mathbf{y}_{i}$ is a corner of order $k$ iff nondom $\left(\left\{\mathbf{y}_{j}^{\kappa} \mid \mathbf{y}_{j} \in \mathcal{Y}\right\}\right)=\mathbf{y}_{i}^{\kappa}$; that is, order $k$ corners are the points that dominate all others in at least one of the $\left(\begin{array}{c}M \\ k\end{array}\right)$ subsets of $k$ objectives.

Clearly, the extremal points in two-criterion sets (e.g., Figures 1 and 3 ) are also corners. However, with more objectives and a finite set, corners defined like this do not correspond to our intuitive notion of where the corners lie. In fact, for the 3-criterion sets shown in Figure 2, the corners of order 1 are the points that minimise single objectives (marked with $\mathbf{\nabla}$ ) and there are no order 2 corners. Note that in the concave case the so-called corners do not lie near to where most people would place the corners.

The reason that there are no order 2 corners is because on projecting onto a pair of criteria there are many non-dominated points; that is $\left|\operatorname{nondom}\left(\left\{\mathbf{y}_{j}^{\kappa} \mid \mathbf{y}_{j} \in \mathcal{Y}\right\}\right)\right|>1$. We therefore arrive at a second definition of edge points.

Definition 2 (Edge Points OF ORder $k$ ): A point $\boldsymbol{y} \in \mathcal{Y}$ is an edge point of order $k$ iff its projection onto $k$ coordinates is not dominated by the projection of any other $\boldsymbol{y}^{\prime} \in \mathcal{Y}$ onto those coordinates.

In fact, as the following theorem shows, these edge points found by projection turn out to be precisely the same points as the attainment surface edge points defined in section 2 and are therefore those illustrated in Figure 4.

THEOREM 1: An attainment surface edge point is equivalent to an edge point order $k$ for some $k$.

PROOF: To see that the non-dominated points following projection onto criterion subsets are the same points as those that extend the range of the attainment surface, suppose that $\mathbf{y}$ is non-dominated when projected onto the criteria $\kappa$; that is, $\mathbf{y}^{\kappa} \in \operatorname{nondom}\left(\left\{\mathbf{y}_{j}^{\kappa} \mid \mathbf{y}_{j} \in\right.\right.$ $\mathcal{Y}\})$. Consequently for criteria $m \in \kappa, y_{m} \leq y_{m}^{\prime}$ for all $\mathbf{y}^{\prime} \in \mathcal{Y}$. Then let $\mathbf{u} \in \mathcal{U} \backslash \mathcal{B}$ be defined as

$$
u_{m}=\left\{\begin{array}{ll}
y_{m} & m \in \kappa \\
b_{m}^{+} & m \notin \kappa
\end{array} \quad m=1, \ldots, M .\right.
$$

By construction $\mathbf{y} \prec \mathbf{u}$. On the other hand, if $\mathbf{y}^{\prime}$ is dominated when projected onto criteria $\kappa$, then there exists an $m \in \kappa$ and $\mathbf{y}^{\prime \prime} \in \mathcal{Y}$ such that $y_{m}^{\prime \prime}<y_{m}^{\prime}$. Therefore, if there is a $\mathbf{u} \in \mathcal{U} \backslash \mathcal{B}$ such that $\mathbf{y}^{\prime} \prec \mathbf{u}$, then $\mathbf{y}^{\prime \prime} \prec \mathbf{u}$, so that $\mathbf{y}^{\prime}$ is not the sole dominator of $\mathbf{u}$. Thus, we have shown that if $\mathbf{y}$ is non-dominated when projected onto indices $\kappa$, then it is the sole dominator of a point $\mathcal{U} \backslash \mathcal{B}$ and is therefore an attainment surface edge point.

Conversely, suppose that $\mathbf{y}$ is an attainment surface edge point. Then there exists a $\mathbf{u} \in \mathcal{U} \backslash \mathcal{B}$ such that $\mathbf{y} \prec \mathbf{u}$, but no other $\mathbf{y}^{\prime} \in \mathcal{Y}$ dominates $\mathbf{u}$. Let $\kappa$ be the indices for which

$$
y_{k} \leq u_{k}<y_{k}^{\prime} \text {. }
$$


Clearly $\kappa$ is not empty because $\mathbf{y} \prec \mathbf{u}$ and $\mathbf{y}^{\prime} \nprec \mathbf{u}$ for all $\mathbf{y}^{\prime} \in \mathcal{Y}\left(\mathbf{y}^{\prime} \neq \mathbf{y}\right)$. Then Equation (9) shows that when projecting onto the criteria $\kappa$, we have $\mathbf{y}^{\kappa} \prec\left(\mathbf{y}^{\prime}\right)^{\kappa}$ for all $\mathbf{y}^{\prime} \in \mathcal{Y}$, which establishes that attainment surface edge points are non-dominated when projected onto some criterion subset.

We remark that it is computationally faster to find edge points by projection onto criterion subsets than using the method described in section 2 .

We anticipate that the equivalence we have shown between points that extend the attainment surface and points that are non-dominated when projected onto some subset of the criteria will be of use in evolutionary multi-objective algorithms (such as Deb et al., 2000; Knowles and Corne, 2000; Zitzler et al., 2002; Smith et al., 2008) which seek to preserve diversity and the spread of the estimated Pareto front by preferentially retaining and perturbing solutions on the periphery of the solution set.

\section{Criterion Subset Edges}

In section 3 we identified edges as the non-dominated points after projection onto $k$ of the $M$ criteria. As we showed there, these points are the points that extend the range of the attainment surface. However, reference to Figure 2 for example shows that points that maximise one of the criteria also lie on the intuitive edges of concave and convex fronts. Here we therefore extend the criterion for a point to be an edge point by including points that, after projection onto $k$ of the criteria, are not dominated if the criteria were to be maximised rather than minimised. A final definition of edge points is thus as follows.

Definition 3 (EDGe Points OF ORder $k$ ): Let $\boldsymbol{y}^{\kappa}$ be the projection of $\boldsymbol{y} \in \mathcal{Y}$ onto $k=|\kappa|$ coordinates. Then $\boldsymbol{y}$ is an order $k$ edge point of $\mathcal{Y}$ iff $\boldsymbol{y}^{\kappa}$ is not dominated under minimisation or not dominated under maximisation by any member $\left\{\boldsymbol{y}_{n}^{k} \mid \boldsymbol{y}_{n} \in \mathcal{Y}\right\}$.

Let the function $\overline{\operatorname{nondom}}(\mathcal{Y})$ be the function which returns the maximal set of nondominated members of $\mathcal{Y}$ under maximisation:

$$
\overline{\operatorname{nondom}}(\mathcal{Y})=\{\mathbf{y} \in \mathcal{Y} \mid \nexists(\mathbf{v} \in \mathcal{Y} \wedge \mathbf{v} \succ \mathbf{y})\} .
$$

The set of edge points of order $k=|\kappa|$ for $\mathcal{Y}$ is therefore

$$
\left\{\mathbf{y}_{n} \mid \mathbf{y}_{n}^{\kappa} \in \operatorname{nondom}\left(\left\{\mathbf{y}_{n}^{\kappa} \mid \mathbf{y}_{n} \in \mathcal{Y}\right\}\right)\right\} \bigcup\left\{\mathbf{y}_{n} \mid \mathbf{y}_{n}^{\kappa} \in \overline{\operatorname{nondom}}\left(\left\{\mathbf{y}_{n}^{\kappa} \mid \mathbf{y}_{n} \in \mathcal{Y}\right\}\right)\right\} .
$$

As an illustration, Figure 5 shows the projection of the hourglass data onto criteria $y_{2}$ and $y_{3}$. Points that are non-dominated under minimisation and maximisation are marked with $\boldsymbol{\nabla}$ and $\boldsymbol{\Delta}$ symbols, respectively. It can be seen that these correspond to different regions of extremal points. The right-hand panel of Figure 5 shows all the edge points identified after projections onto all the criterion subsets. As the figure illustrates, this definition of edge points has identified a uniform spread of points corresponding to what we intuitively identify as edges across both concave and convex portions of the front.

The attainment surface of $\mathcal{Y}$ under maximisation, $\overline{\mathcal{S}}$, may be defined as the boundary of the region dominated under maximisation by the elements of $\mathcal{Y}$. Figure 6 illustrates the attainment surfaces under minimisation and maximisation for a set of mutually nondominating points in the plane. Whereas the attainment surface under minimisation is a conservative interpolation of the set (Smith et al., 2008) in the sense that every point in $\mathcal{S}$ is weakly dominated by an element of $\mathcal{Y}$, the attainment surface under maximisation can be seen to be an optimistic interpolation of $\mathcal{Y}$. Using the same arguments as presented in 

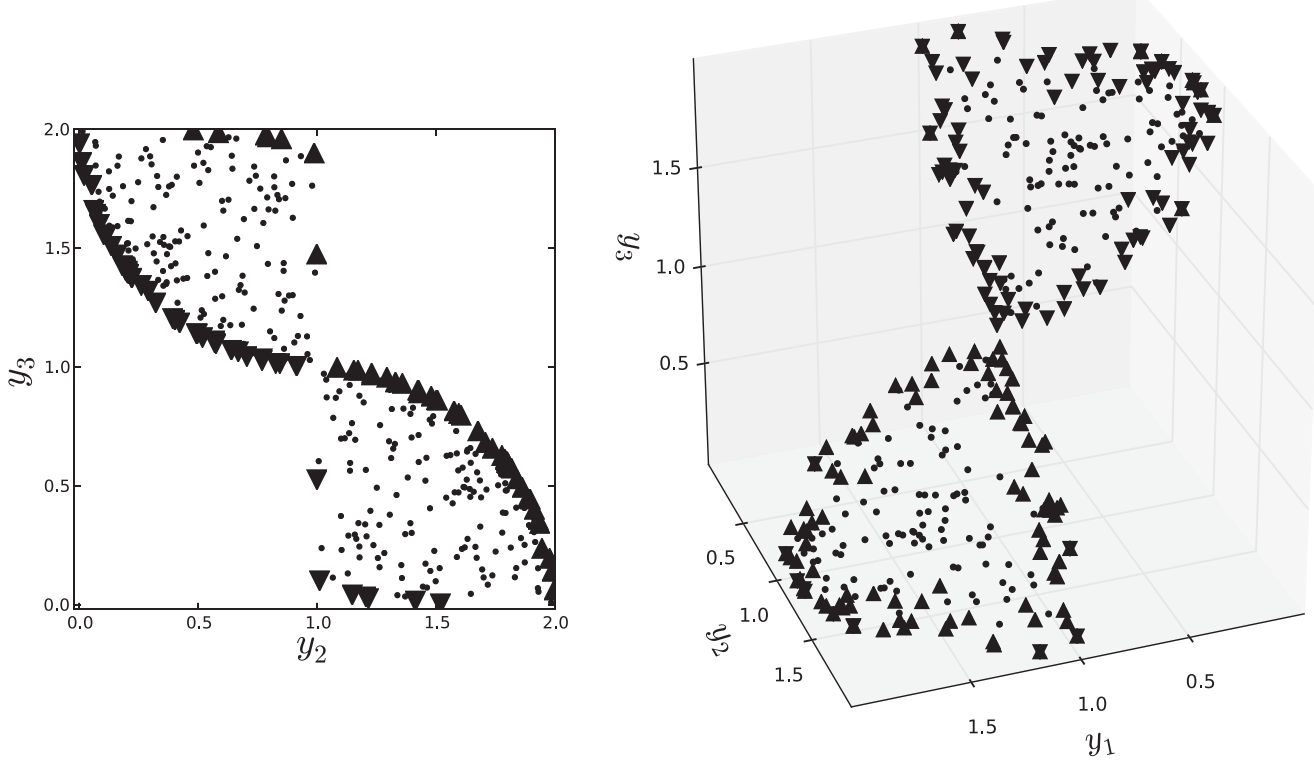

Figure 5: Edge points by projection. Left: The 3D hourglass data projected onto criteria $y_{2}$ and $y_{3}$. Right: Edge points of the hourglass data identified as non-dominated under minimisation or maximisation after projection onto criterion subsets. In both panels, points that are non-dominated under minimisation and maximisation are marked with $\boldsymbol{\nabla}$ and $\boldsymbol{\Delta}$ symbols, respectively.

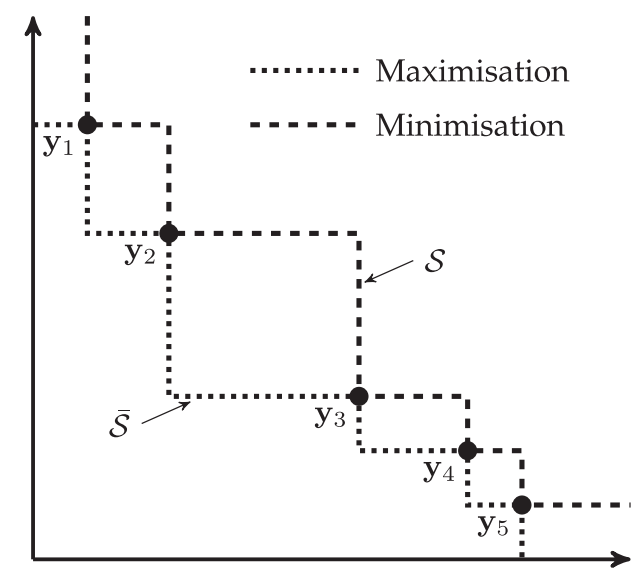

Figure 6: Attainment surfaces of a mutually non-dominating set of points $\mathcal{Y}=\left\{\mathbf{y}_{i}\right\}$ under minimisation and maximisation.

section 3 , the edge points identified as non-dominated under maximisation are exactly those that extend the range of the attainment surface under maximisation. We note that for two criteria, the extremal points are edge points because they extend both $\mathcal{S}$ and $\overline{\mathcal{S}}$, but of course, this is not the case with more than two criteria. 

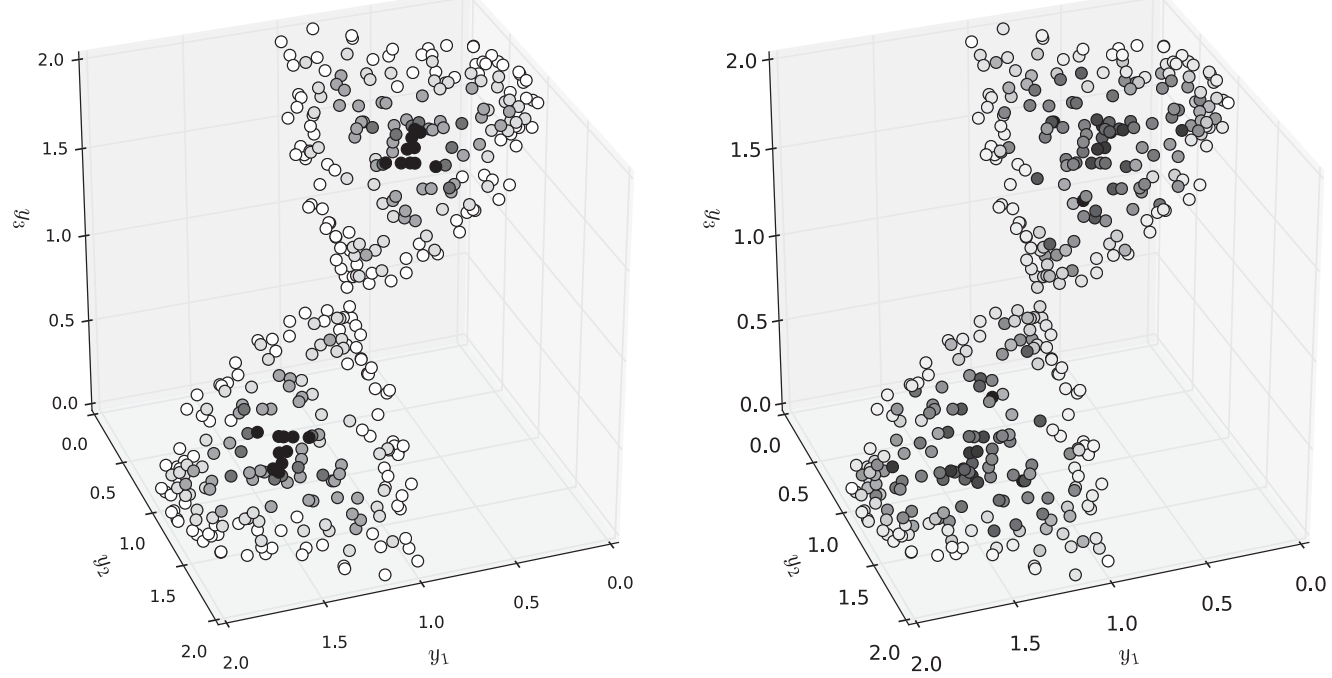

Figure 7: Quantifying edginess. The 3D hourglass data with shaded points according to how close a point is to being an edge point. Left: Identified edge points are removed from the set to leave a reduced set of mutually non-dominating points from which "second degree" edges can be identified. This procedure is repeated iteratively until no points remain. Points are shaded according to the iteration at which they are identified as edges. Right: As described in Section 6, points are ordered by the largest $s$ parameter in the CDAS mapping for which they are CDAS edge points.

\section{Using Edges as Landmarks in Visualisation}

Having defined edges, we now examine their use in the visualisation of many objective mutually non-dominating sets such as those resulting from many objective optimisations.

In addition to knowing which are the edge points, it may also be useful to know which are the interior points in the sense that interior points are far from being on the edges. A straightforward procedure for assigning a degree of "edginess" to points is the following deflationary procedure. First, identify the edge points of the set $\mathcal{E}_{1}=\operatorname{edge}(\mathcal{Y})$, where edge $(\cdot)$ is the function that returns the set of edge points of its argument. The edge points are then removed from $\mathcal{Y}$ and second degree edges are found: $\mathcal{E}_{2}=\operatorname{edge}\left(\mathcal{Y} \backslash \mathcal{E}_{1}\right)$. This procedure is repeated until all the points have been removed. Figure 7 shows the result of this procedure applied to the $3 \mathrm{D}$ hourglass dataset. It is clear that points near to the intuitive centre of the set have been located. It is likely that these will be of interest to decision makers because, given the choices available in the set, their central position means that they do not favour any particular criterion. We note that this procedure assigns to each point an integer degree of edginess from a usually small number of degrees. Thus, many points have the same degree of edginess, so this procedure does not provide a total ordering of the points. We provide an alternative measure of the degree of edginess in Section 6.

Hughes $(2007 \mathrm{a}, 2007 \mathrm{~b})$ proposed as a prototypical many-objective problem the design of a pulsed doppler radar to simultaneously measure the velocity and distance of a target. He applied the MSOPS algorithm (Hughes, 2005) to the optimisation an 
appropriate set of waveforms that will maximise the detection performance and minimise the duration of the transmission. The problem has nine objectives: objectives 1 , 3,5 , and 7 are concerned with the range at which the object may be detected, while objectives 2, 4, 6, and 8 relate to its velocity; objective 9 is the total transmission time of the waveform that is being optimised. The data we use is arranged so that all the objectives are to be minimised.

To visualise the edges of the radar dataset, we first project it into two dimensions. Rather than use the objective values directly, we seek to capture the order relations between the elements of the set. This is done by defining a dominance distance between elements that quantifies the extent to which two elements are on average greater than, less than, or equal to other elements in the set. More specifically, we define a similarity between two elements $\mathbf{y}_{m}$ and $\mathbf{y}_{n}$ relative to the element $\mathbf{y}_{p}$ as follows:

$$
\begin{aligned}
S\left(\mathbf{y}_{m}, \mathbf{y}_{n} ; \mathbf{y}_{p}\right)= & \sum_{j=1}^{M} \gamma_{j}\left[I\left(\left(y_{p j}<y_{m j}\right) \wedge\left(y_{p j}<y_{n j}\right)\right)\right. \\
& +I\left(\left(y_{p j}=y_{m j}\right) \wedge\left(y_{p j}=y_{n j}\right)\right) \\
& \left.+I\left(\left(y_{p j}>y_{m j}\right) \wedge\left(y_{p j}>y_{n j}\right)\right)\right]
\end{aligned}
$$

where $I(p)$ is 1 if $p$ is true and 0 otherwise. Thus the dominance similarity is the weighted number of objectives on which $\mathbf{y}_{m}$ and $\mathbf{y}_{n}$ are both less than, greater than, or equal to $\mathbf{y}_{p}$. The weights $\gamma_{j}\left(\gamma_{j} \geq 0\right.$ and $\left.\sum_{j}^{M} \gamma_{j}=1\right)$ reflect the importance assigned to each objective; here we set them all equal: $\gamma_{j}=1 / M$. The dominance distance between $\mathbf{y}_{m}$ and $\mathbf{y}_{n}$ is then defined by averaging over all the other elements in the set:

$$
D\left(\mathbf{y}_{m}, \mathbf{y}_{n}\right)=1-\frac{1}{N-2} \sum_{p \notin\{m, n\}} S\left(\mathbf{y}_{m}, \mathbf{y}_{n} ; \mathbf{y}_{p}\right) .
$$

The dominance distance is formally a metric (Walker et al., 2013) and can be efficiently calculated from

$$
D\left(\mathbf{y}_{m}, \mathbf{y}_{n}\right)=\sum_{j=1}^{K} \gamma_{j}\left(\left|r_{m j}-r_{n j}\right|-1\right)
$$

where $r_{m j}$ is the rank of $\mathbf{y}_{m}$ on criterion $j$. With this measure of the distance between elements of the set, metric multi-dimensional scaling (MDS; Sammon, 1969; Webb, 2002) is then used to find an embedding in Euclidean space that preserves the dominance distances. Figure 8 shows the dominance distance MDS visualisation of 200 solutions of the radar data, in which solutions are coloured by their average rank. Also shown are the solutions which minimise or maximise a single objective, which are the order 1 edges. As the figure shows, the visualisation provides a topographic low-dimensional representation of the data and the order 1 edges tend to lie around the periphery of the points plotted in the plane. Additional details and visualisations are given by Walker et al. (2013).

The relative sparsity of points in many dimensions and the decreasing discriminatory power of the dominance relation with increasing dimension means that with many objectives most points in a many-objective mutually non-dominating set are edge points. Figure 9 shows the variation with $k$ of the fraction of 2,000 radar data points that are identified as edges according to maximisation or minimisation. Clearly, the fraction 


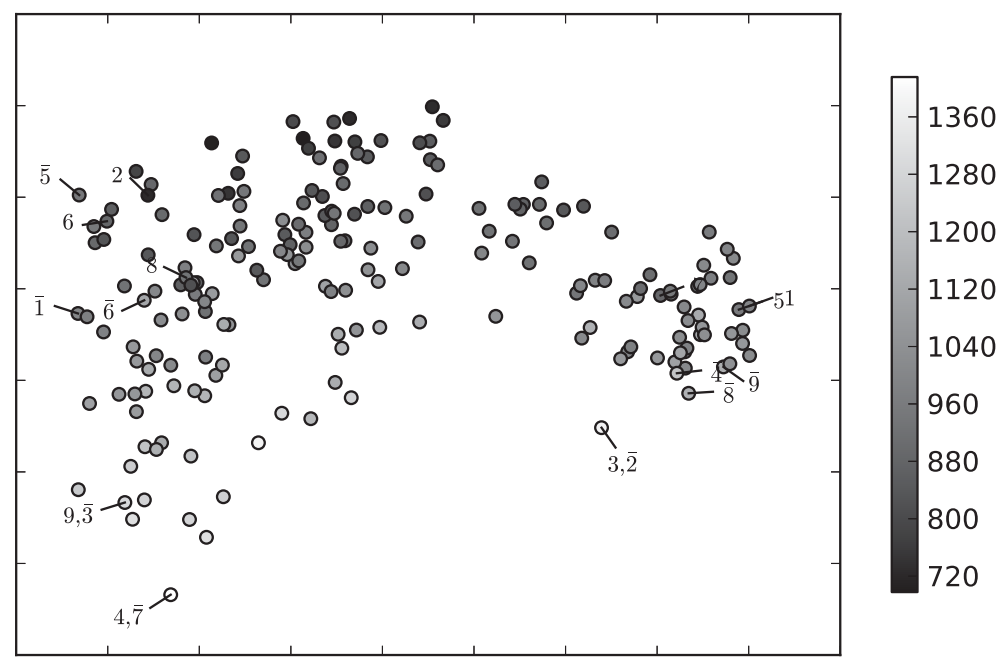

Figure 8: Dominance distance MDS visualisations of 200 samples from the radar data. The greyscale indicates the average rank of the solution; solutions that maximise or minimise a single objective are indicated with the number of the objective; if the objective is maximised the number is indicated with an overbar.
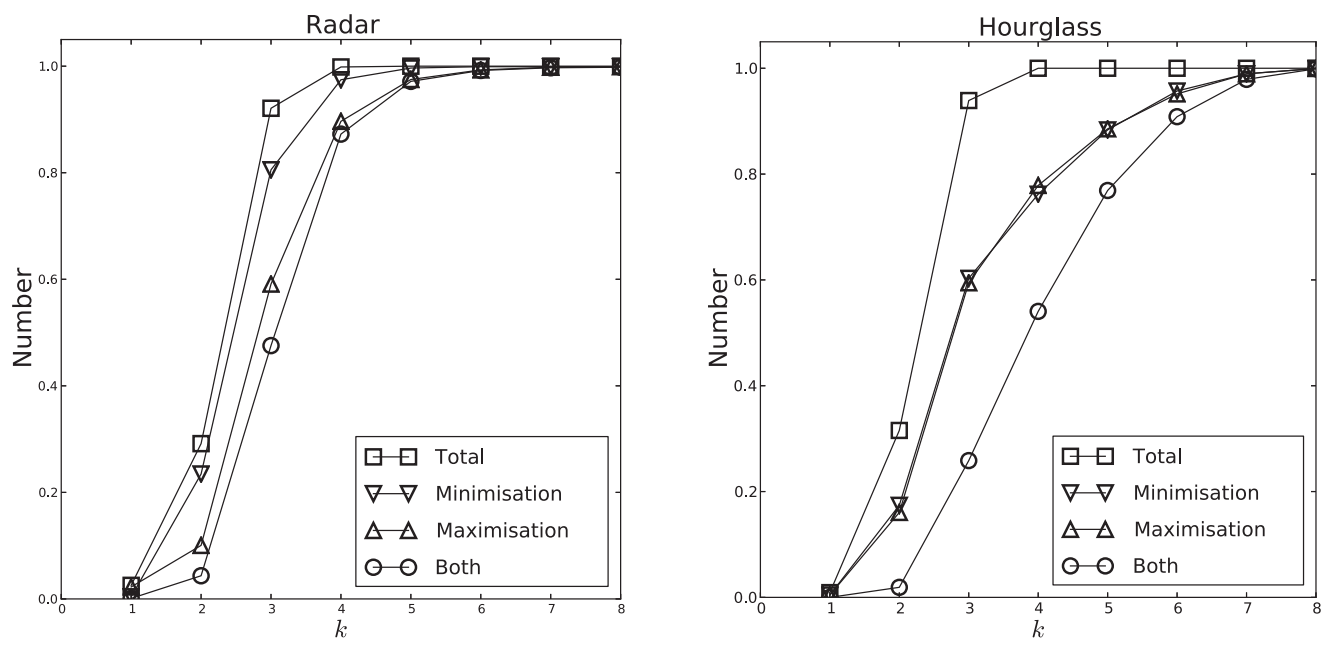

Figure 9: The fraction of order $k=|\kappa|$ edge points in 2,000 solutions from the radar estimated Pareto front and from a nine-criterion hourglass mutually non-dominating set. Plots show the number of edge points arising from non-dominance under minimisation, under maximisation, together with the total number of edge points (the union of the minimisation and maximisation sets) and the number that are non-dominated under both minimisation and maximisation (the intersection of the minimisation and maximisation sets).

of edge points grows rapidly with the number of criteria. The figure also shows the fraction of order $k$ edge points for 2,000 points uniformly distributed on a 9-dimensional hourglass dataset. We note that here the fraction of edge points identified by minimisation and maximisation is very similar and arises because of the symmetrical concave 
and convex regions of the hourglass data. For the radar data, in contrast, the number of edge points corresponding to minimisation considerably exceeds the number arising from maximisation. By comparison with Figure 4 and calculations for entirely convex and concave fronts, we therefore infer that the radar data is on the whole more concave (like Figure 4(a)) than convex (like Figure 4(b)), although it may have separate concave and convex regions.

For any $k$, a point may be an edge point in $\left(\begin{array}{c}M \\ k\end{array}\right)$ ways considering minimisation and the same number considering maximisation. The $k$-preference ordering also considers the projection of a mutually non-dominating set onto combinations of the coordinate axes (Das and Dennis, 1998; di Pierro et al., 2007). A point is said to be efficient of order $k$ if it is not dominated (under minimisation) in projections onto any of the $\left(\begin{array}{c}M \\ k\end{array}\right)$ criterion subsets. We therefore consider the fraction of the $\left(\begin{array}{c}M \\ k\end{array}\right)$ projections for which a point is nondominated. Clearly, as this fraction approaches 1 , the edge point becomes $k$-efficient. Figure 10 shows the dominance distance embedding of the radar data divided into cells, each of which is shaded according to the median (over points in the cell) of this fraction. We show visualisations for $k=3,5$, and 7, but results for other $k$ are similar. As shown, edge points from both minimisation and maximisation tend to occur more frequently close to the edges of the dominance distance MDS projection. The projection and this definition of edge points therefore concur about where the boundaries of the set lie.

We note that efficient edge points for all $k$ arising from maximisation tend to occur close to the horns of the crescent, which is where points maximising individual criteria are mostly located, as shown in Figure 8. Likewise, points arising from minimisation tend to be located near to the top of the visualisation, where points minimising individual criteria are mostly located.

For comparison, Figure 11 shows the dominance distance MDS embedding of ninedimensional hourglass data, again shaded according to the median fractional efficiency of edge points for $k=6$; similar pictures are obtained with other $k$. The MDS embedding of the hourglass data comprises two orthogonal 2D-plates, corresponding to the concave and convex regions of the data, and thus requires three dimensions for visualisation. Figure 11 therefore shows the projections onto the three most significant axes. Here it is evident that although with nine-dimensional data almost all points are edge points, those obtained by non-domination under maximisation lie principally on one plate (the convex plate), while edge points identified by minimisation lie on the other plate (cf. Figure 5). Returning to the radar data, we infer that set is approximately convex in the vicinity of the crescent's horns, but more concave close to the other boundary.

An additional interesting feature revealed by this visualisation of the edge points is the region of higher edge point density crossing the embedding from top to bottom toward the right-hand third of the embedding. This is particularly evident in the lefthand panel of Figure 12, which shows the median efficiency of $k=6$ edge points from either maximisation or minimisation; other $k$ yield similar pictures. The criteria on which the radar data is optimised divide into three groups. One group $\left(f_{1}, f_{3}, f_{5}\right.$, and $\left.f_{7}\right)$ relates to the range at which a radar can discern targets. The second group $\left(f_{2}, f_{4}\right.$, $f_{6}$, and $f_{8}$ ) relates to the speed at which moving targets can be detected, while the final group $\left(f_{9}\right)$ comprises a single objective, the transmission time of the radar waveform. Individual solutions tend to optimise one of these groups of criteria at the expense of the others (Walker et al., 2013). Plotting the solutions with symbols showing which of these groups is best optimised, Figure 12 shows that the high edge point density region corresponds to the transition between solutions that best optimise the range objectives and those that best optimise the velocity objectives. 

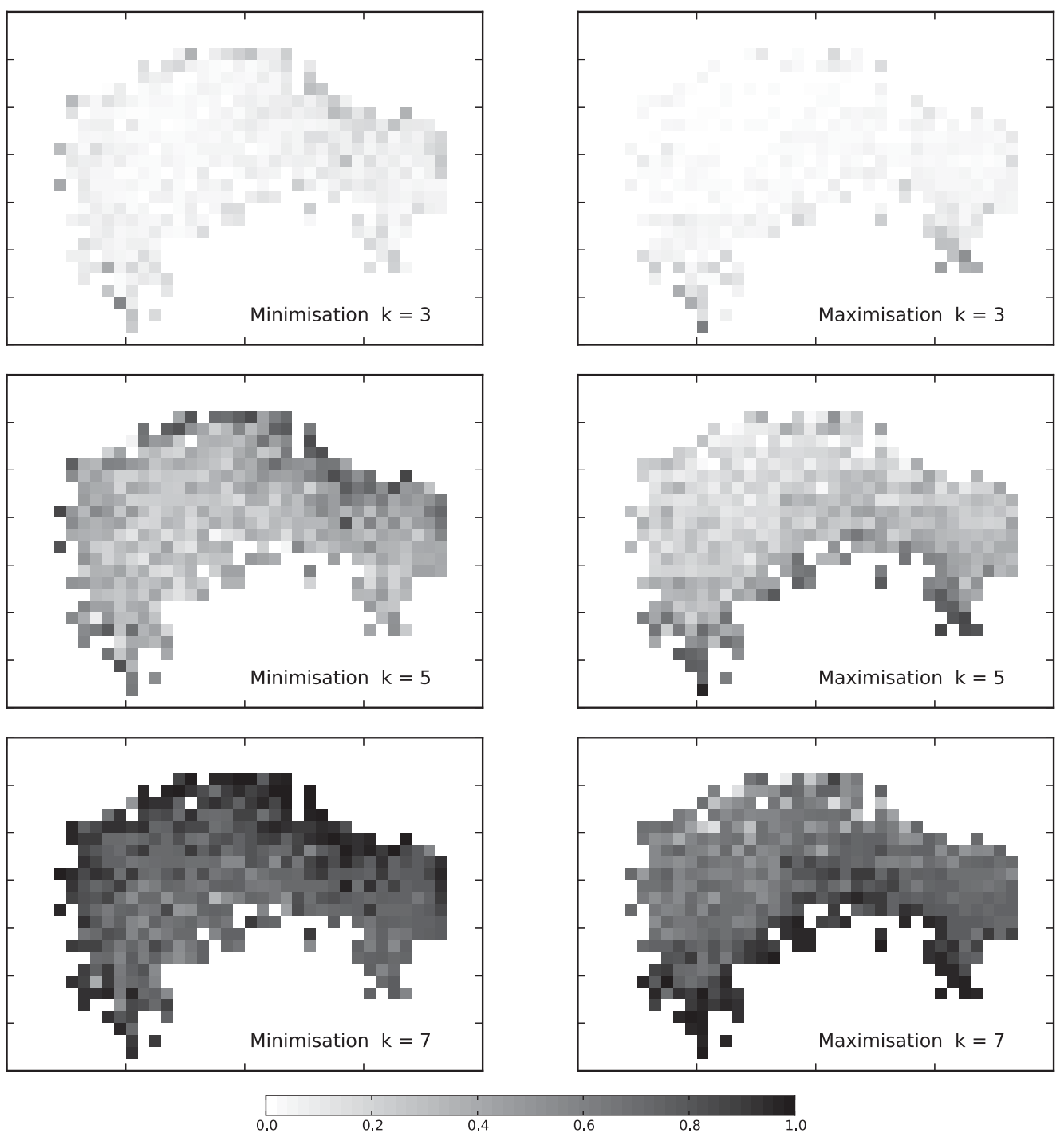

Figure 10: Cellular visualisations of the radar population embedded into two dimensions with dominance distance MDS. The greyscale indicates the the median fractional efficiency with which edge points in the cell are edge points in a $k$-criterion subset. Panels in the left- and right-hand columns arise from minimisation and maximisation, respectively.

\section{Controlling Dominance Area of Solutions}

As we have seen, as the number of objectives increases most elements of a mutually nondominating set are edge points. In addition to the sparsity of points in high-dimensional spaces, the dominance relation becomes less discriminatory as the number of criteria increases. A number of methods have been proposed that attempt to strengthen the dominance relation in many dimensions, or otherwise provide additional resolution to the ordering generated from Pareto dominance (Bentley and Wakefield, 1997; Drechsler 

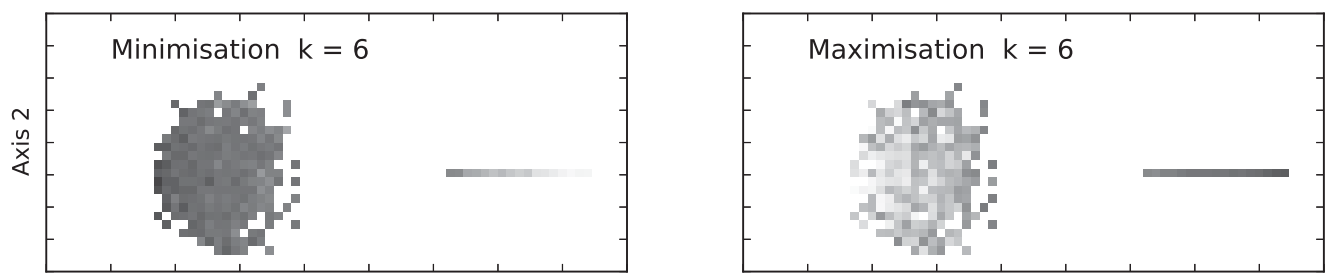

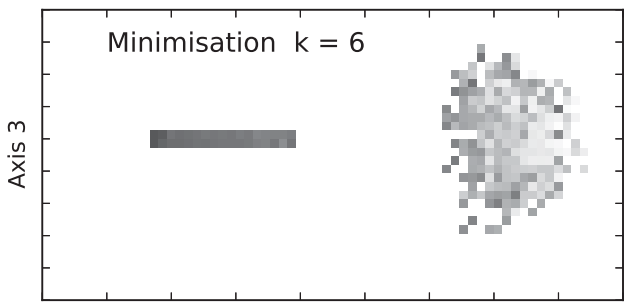

Axis 1

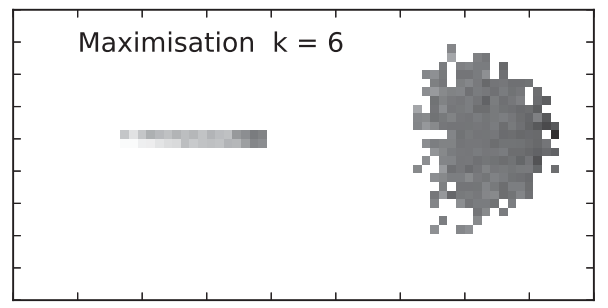

Axis 1

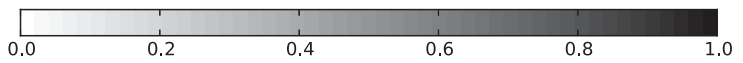

Figure 11: Median fractional efficiency of edges points of order 6 in a dominance distance MDS embedding of the nine-dimensional hourglass data. Edges arising from minimisation and maximisation are shown on the left- and right-hand columns, respectively. Projections onto MDS axes 1 and 2, and axes 1 and 3, are shown in the two rows.

et al., 2001; Laumanns et al., 2002; di Pierro et al., 2007; Sato et al., 2007a; Emmerich et al., 2013). Here we examine the use of the controlling dominance area of solutions (CDAS; Sato et al., 2007a) approach, which has been successfully used in some recent manyobjective optimisers (Sato et al., 2007b, 2010; de Carvalho and Pozo, 2011; Woolard and Fieldsend, 2013).

The region dominated by a point is bounded by planes that intersect the coordinate axes at right angles. In the CDAS scheme, a point dominates the region bounded by planes that make an angle $\phi$ with the coordinate axes. When $\phi<\pi / 2$, the dominance relation is strengthened in the sense that a point dominates a larger volume than when $\phi=\pi / 2$; conversely, when $\phi>\pi / 2$, a point dominates a smaller volume, effectively weakening the dominance relation. A particularly attractive feature of the CDAS dominance is that it may be implemented by nonlinearly mapping points to a new space and then using the usual dominance relation. The mapping $\mathbf{z}=\mathbf{g}(\mathbf{y})$ is defined by:

$$
g_{m}(\mathbf{y} ; \mathbf{s})=\|\mathbf{y}\| \frac{\sin \left(\omega+s_{m} \pi\right)}{\sin \left(s_{m} \pi\right)} \quad m=1, \ldots, M
$$

where $\omega=\arccos \left(y_{m} /\|\mathbf{y}\|\right)$ and $\mathbf{s}$ is a vector of parameters defining the angles that the planes forming the dominating plane make with the coordinate axes, $\phi_{m}=\pi s_{m}$. Here, in common with other work, we use the same $s_{m}$ for all coordinates: $s_{m}=s$ for all $m$. When $s=1 / 2$, conventional dominance is obtained. The weakest dominance relation is obtained when $s=0.75$, which corresponds in the mapped space to all the elements of the set being mapped to a simplex; while when $s=0.25$, the points are mapped to a ray passing through the origin and the points are totally ordered.

To define CDAS edge points, we first select a $k$-dimensional subset of objectives $\mathbf{y}^{k}$, which are then mapped using the CDAS transformation, and then locate the edges of 

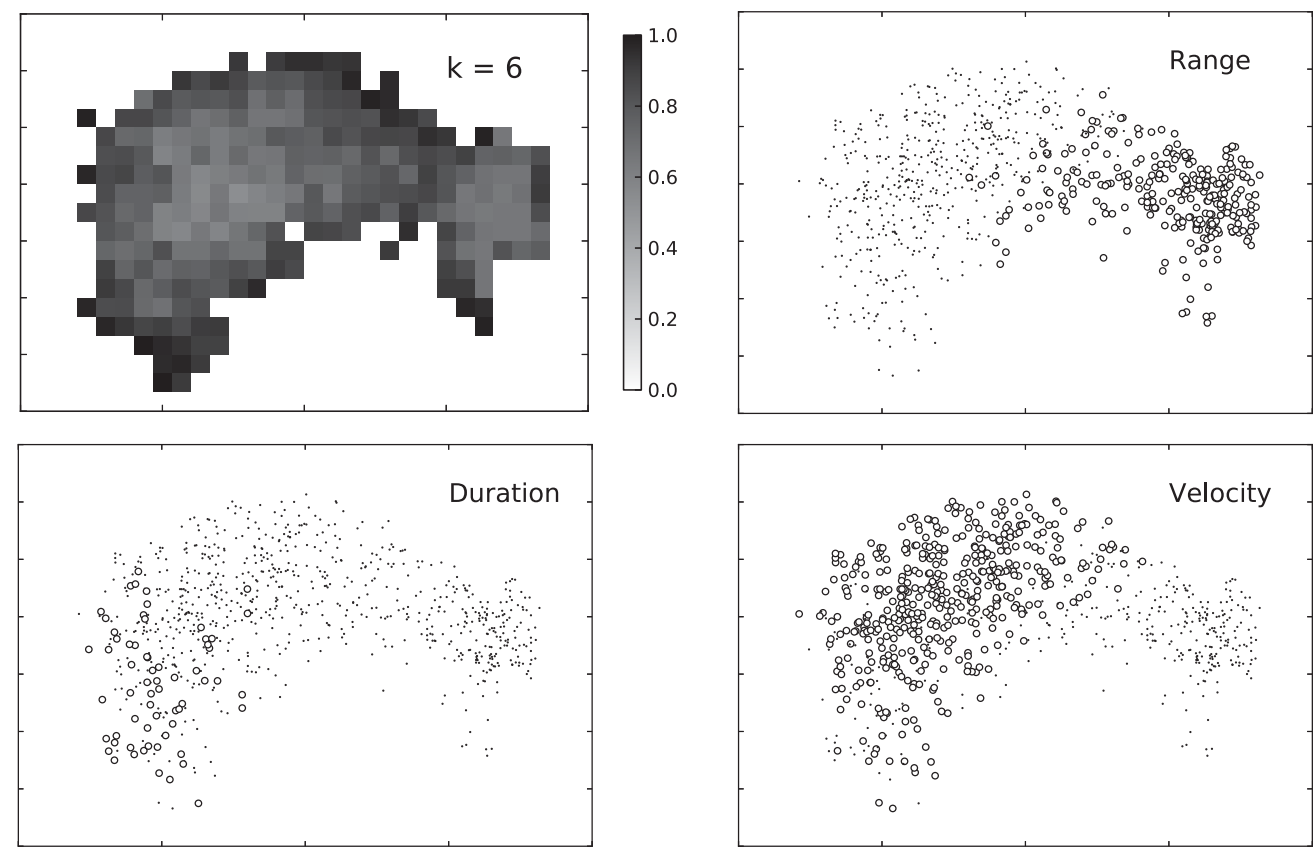

Figure 12: Top left: Median fractional efficiency of edge points of order 6 in a dominance distance MDS embedding of the radar data. Remaining figures: Dominance distance embedding of the radar data solutions with symbols indicating the type of objective for which the solution is most highly ranked. Solutions marked with a circle belong to the indicated group of objective (range, velocity or duration). The transition between solutions that best optimise range criteria and those that best optimise velocity criteria or transmission time lies in the same region as the high density of efficient edge points shown in the top left panel.

the mapped set $\mathcal{Z}=\left\{\mathbf{g}\left(\mathbf{y}^{\kappa}\right) \mid \mathbf{y} \in \mathcal{Y}\right\}$ using conventional dominance in the mapped space. A point $\mathbf{y} \in \mathcal{Y}$ is thus defined as a CDAS edge point if the image under $\mathbf{g}$ of a subset of its coordinates is an edge point of $\mathcal{Z}$.

Figure 13 shows CDAS edge points for the 3D hourglass dataset for $s=0.35, s=$ 0.5 (conventional dominance), and $s=0.7$. When $s=0.7$, the dominance relation is weakened and there is consequently a thicker band of edge points extending inward from the intuitive edges of the set. In the limit $s \rightarrow 0.75$, all points are edge points. On the other hand, when $s<0.5$, the stronger dominance relation implies that fewer points define the attainment surface of $\mathcal{Z}$ (that is, are non-dominated on projection onto criterion subsets ${ }^{1}$ ) so that a sparser set of stronger edge points is identified. In the limit $s \rightarrow 0.25$, the number of minimising or maximising edge points is each equal to $M$, the number of criteria. However, these CDAS edge points do not necessarily coincide with the order 1 edge points using conventional dominance.

\footnotetext{
${ }^{1}$ Viewed in the original space, the projection is no longer an orthogonal projection along particular coordinates.
} 


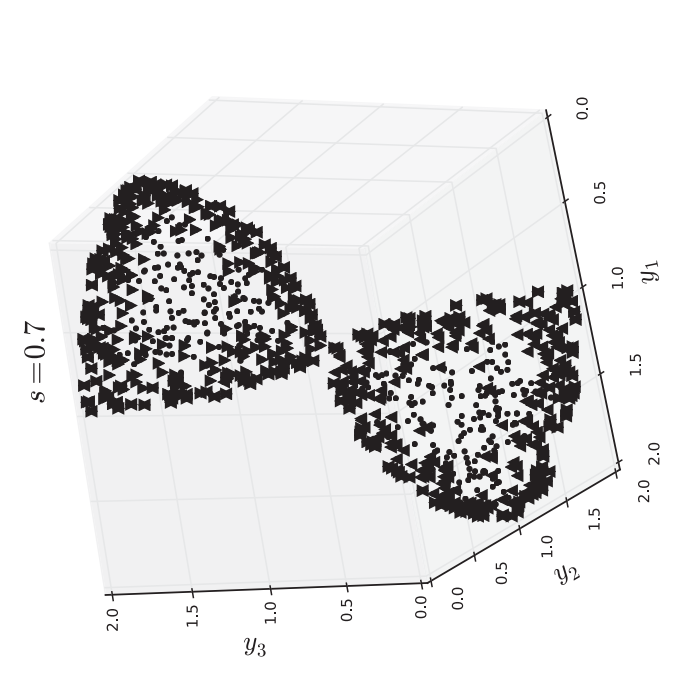

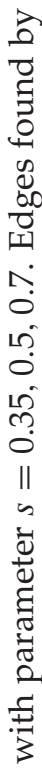
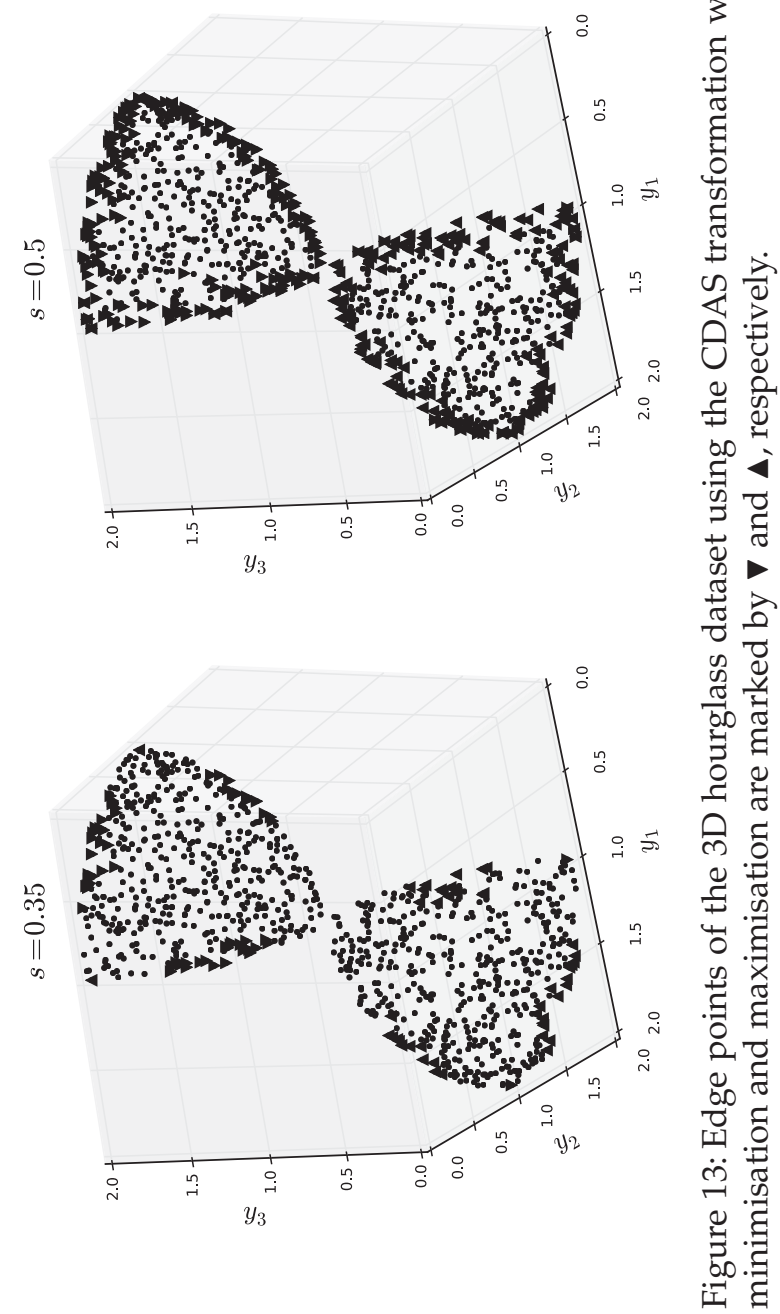

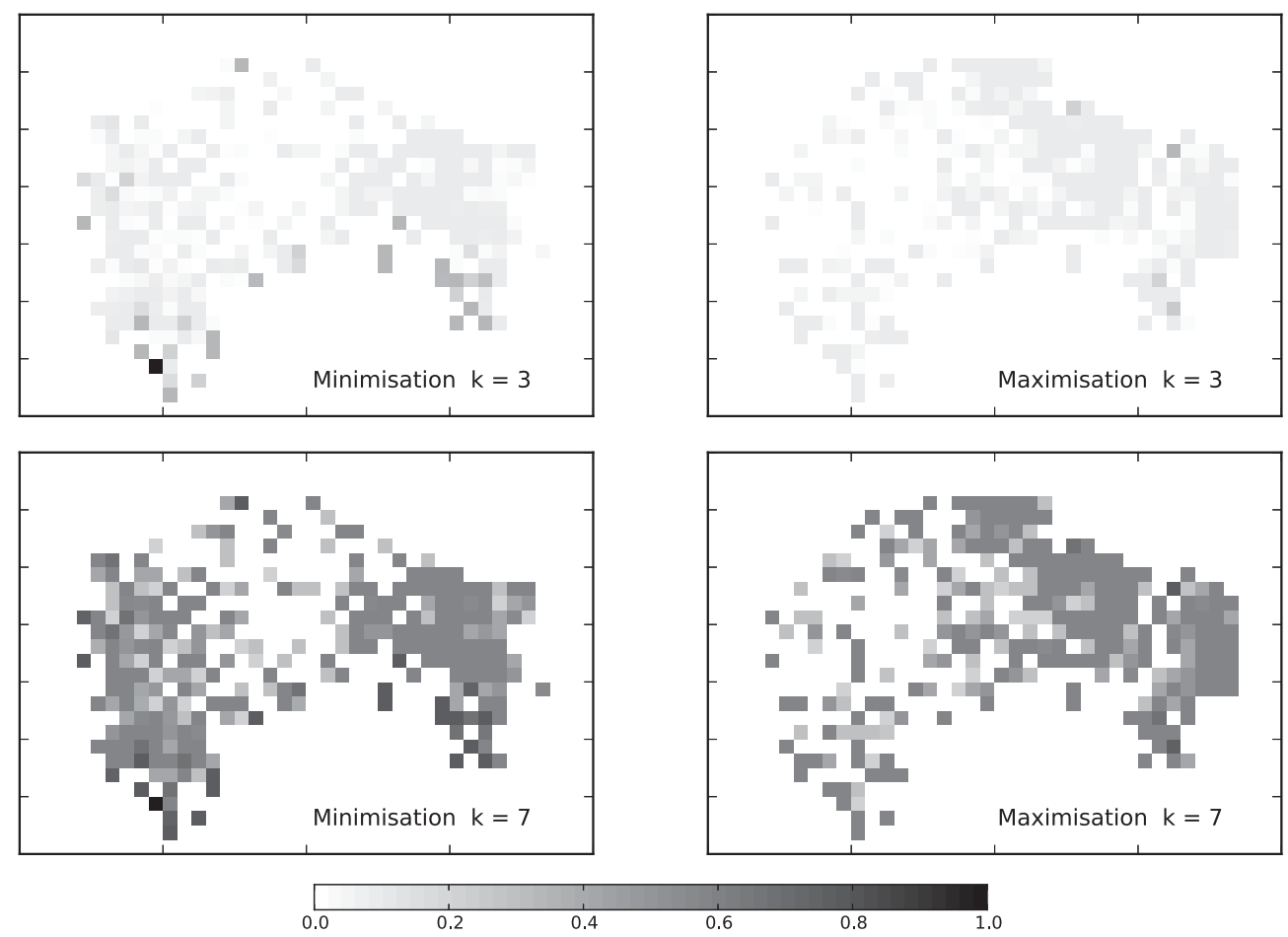

Figure 14: CDAS edge points of the radar dataset using the CDAS transformation with parameter $s=0.26$ for orders $k=3,7$. Median fractional efficiency of edges in cells arising from minimisation and maximisation are shown on the left- and right-hand columns, respectively.

In Section 5 we described a method of assigning a degree of edginess to points by successively removing edge points from a set. An alternative measure of the degree of edginess can be obtained as the maximum $s$ for which a point is an edge point. (It is not hard to see that if a point is a CDAS edge point for some $s$, then it is also an edge point for all $s^{\prime}>s$.) Unlike the deflationary method, this measure assigns the degree of edginess on a continuous scale and thus generally totally orders the points-and has previously been used to rank solutions in a many-objective optimiser (Woolard and Fieldsend, 2013). In addition, it can be used for high dimensional sets for which the deflationary method fails because all points are edge points. Figure 7 shows that applying this measure to a 3D hourglass dataset identifies the interior regions of the set.

Figure 14 shows the median fractional efficiency of edge points for the radar data, using $s=0.26$. This illustrates how using a small $s$ effectively reduces the number of edge points in high dimensions. In the nine-dimensional hourglass data, as here, we find that the strongest edge points are generally close to the periphery of the visualisation.

\section{Conclusion}

We have explored a number of ways of extending the simple idea of the extreme elements of a mutually non-dominating set to more than two criteria. Our definitions 
of edge points are closely related to the attainment surface, which plays a fundamental role in understanding which points lie close to the edges of a mutually non-dominating set. We have shown that the definition of edge points as those points that extend the range of the attainment surface yields precisely the points that are non-dominated in a projection onto a subset of the criteria.

These definitions of edge points are closely related to preference ordering, in which a many-objective point is considered efficient if it remains non-dominated when projected onto all possible combinations of $k$ criterion subsets. A measure, therefore, of the strength of edge points is the fraction of the possible combinations in which they remain nondominated, and we note that di Pierro et al. (2007) employed this idea for many-objective optimisation.

Although somewhat counterintuitive, we have shown that points that remain nondominated under maximisation after projection onto criterion subsets are also important, because they too are on the edges of the set. They play a particularly important role in convex-shaped sets and we anticipate that using edge points from maximisation will be important in maintaining diversity in many-objective optimisation algorithms.

As the number of criteria increases and almost all points are edge points and thus extend the range of the attainment surface, we emphasise that regions with a high frequency of edge points and edge points that are non-dominated in many projections correspond to the boundaries of low-dimensional visualisations, and furthermore reveal previously unknown structure in a many-objective optimisation problem.

The edge points of a set retain a useful meaning using the modified CDAS dominance relation. This provides another method of identifying strong edge points and of locating points that lie in the interior of the set.

Software implementing these ideas and an IPython notebook (Pérez and Granger, 2007) showing the generation of colour versions of the figures is available at https:// github.com/richardeverson/life-on-the-edge.

\section{References}

Bentley, P., and Wakefield, J. (1997). Finding acceptable solutions in the Pareto-optimal range using multiobjective genetic algorithms. Soft Computing in Engineering Design and Manufacturing, 5:231-240.

Das, I., and Dennis, J. E. (1998). Normal-boundary intersection: A new method for generating the Pareto surface in nonlinear multicriteria optimization problems. SIAM Journal on Optimization, 8(3):631-657.

de Carvalho, A. B., and Pozo, A. (2011). Using different many-objective techniques in particle swarm optimization for many objective problems: An empirical study. International Journal of Computer Information Systems and Industrial Management Applications, 3:96-107.

Deb, K., Agrawal, S., Pratab, A., and Meyarivan, T. (2000). A fast elitist non-dominated sorting genetic algorithm for multi-objective optimization: NSGA-II. KanGAL report 200001, Indian Institute of Technology, Kanpur, India.

di Pierro, F., Khu, S. T., and Savic, D. (2007). An investigation on preference ordering ranking scheme in multiobjective evolutionary optimization. IEEE Transactions on Evolutionary Computation, 11(1):17-45.

Drechsler, N., Drechsler, R., and Becker, B. (2001). Multi-objective optimisation based on relation favour. In Proceedings of the Evolutionary Multi-Criterion Optimization Conference, pp. 154-166. Berlin: Springer. 
Emmerich, M., Deutz, A., Kruisselbrink, J., and Shukla, P. K. (2013). Cone-based hypervolume indicators: Construction, properties, and efficient computation. In Proceedings of the Evolutionary Multi-Criterion Optimization Conference, pp. 111-127.

Everson, R. M., Walker, D. J., and Fieldsend, J. E. (2013). Edges of mutually non-dominating sets. In Proceedings of the Genetic and Evolutionary Computation Conference, GECCO 2013, pp. 607-614.

Farina, M., and Amato, P. (2003). Fuzzy optimality and evolutionary multiobjective optimization. In Proceedings of the Evolutionary Multi-Criterion Optimization Conference, EMO 2003, pp. 72-73.

Fieldsend, J. E., and Everson, R. M. (2013). Visualising high-dimensional Pareto relationships in two-dimensional scatterplots. In Proceedings of the Evolutionary Multi-Criterion Optimisation Conference, EMO 2013, pp. 558-572.

Hughes, E. J. (2005). Evolutionary many-objective optimisation: Many once or one many? In Proceedings of the IEEE Congress on Evolutionary Computation, CEC 2005, pp. 222-227.

Hughes, E. J. (2007a). Best known non-dominated results of radar waveform optimisation. Available at code.evanhughes.org.

Hughes, E. J. (2007b). Radar waveform optimisation as a many-objective application benchmark. In Proceedings of the Evolutionary Multi-criterion Optimization Conference, EMO 2007, pp. 700-714.

Ishibuchi, H., Tsukamoto, N., and Nojima, Y. (2008). Evolutionary many-objective optimization: A short review. In Proceedings of the IEEE Congress on Evolutionary Computation, CEC 2008, pp. 2419-2426.

Knowles, J. D., and Corne, D. W. (2000). Approximating the nondominated front using the Pareto archived evolution strategy. Evolutionary Computation, 8(2):149-172.

Kudo, F., and Yoshikawa, T. (2012). Knowledge extraction in multi-objective optimization problem based on visualization of Pareto solutions. In Proceedings of the IEEE Congress on Evolutionary Computation, CEC 2012, pp. 860-865.

Laumanns, M., Thiele, L., Deb, K., and Zitzler, E. (2002). Combining convergence and diversity in evolutionary multi-objective optimization. Evolutionary Computation, 10(3):263-282.

Obayashi, S. (2002). Pareto solutions of multipoint design of supersonic wings using evolutionary algorithms. In Proceedings of the Adaptive Computing in Design and Manufacture V Conference, pp. $3-15$.

Pérez, F., and Granger, B. E. (2007). IPython: A system for interactive scientific computing. Computing in Science and Engineering, 9(3):21-29.

Sammon, J. W. (1969). A nonlinear mapping for data structure analysis. IEEE Transactions on Computers, 18(5):401-409.

Sato, H., Aguirre, H., and Tanaka, K. (2007a). Controlling dominance area of solutions in multiobjective evolutionary algorithms and performance analysis on multiobjective $0 / 1$ knapsack problems. IPSJ Digital Courier, 3:703-718.

Sato, H., Aguirre, H. E., and Tanaka, K. (2007b). Controlling dominance area of solutions and its impact on the performance of MOEAs. In Proceedings of the Evolutionary Multi-Criterion Optimization Conference, EMO 2007, pages 5-20.

Sato, H., Aguirre, H. E., and Tanaka, K. (2010). Self-controlling dominance area of solutions in evolutionary many-objective optimization. In Simulated Evolution and Learning (pp. 455-465). Berlin: Springer.

Singh, H., Isaacs, A., and Ray, T. (2011). A Pareto corner search evolutionary algorithm and dimensionality reduction in many-objective optimization problems. IEEE Transactions on Evolutionary Computation, 15(4):539-556. 
Smith, K., Everson, R., Fieldsend, J., Misra, R., and Murphy, C. (2008). Dominance-based multiobjective simulated annealing. IEEE Transactions on Evolutionary Computation, 12(3):323-343.

Tušar, T., and Filipič, B. (2011). Visualizing 4D approximation sets of multiobjective optimizers with prosections. In Proceedings of the Genetic and Evolutionary Computation Conference, GECCO 2011, pp. 737-744.

Walker, D., Everson, R., and Fieldsend, J. (2013). Visualising mutually non-dominating solution sets in many-objective optimisation. IEEE Transactions on Evolutionary Computation, 17(2):165-184.

Webb, A. R. (2002). Statistical pattern recognition, 2nd ed. New York: Wiley.

Woolard, M. M., and Fieldsend, J. E. (2013). On the effect of selection and archiving operators in many-objective particle swarm optimisation. In Proceedings of the Genetic and Evolutionary Computation Conference, GECCO 2013, pp. 129-136.

Yoshikawa, T., Yamashiro, D., and Furuhashi, T. (2007). A proposal of visualization of multiobjective Pareto solutions-Development of mining technique for solutions. In Proceedings of the 2007 IEEE Symposium on Computational Intelligence in Multicriteria Decision Making (MCDM 2007), pp. 172-177.

Zitzler, E. (1999). Evolutionary algorithms for multiobjective optimization: Methods and applications. Ph.D. thesis, ETH Zurich.

Zitzler, E., Laumanns, M., and Thiele, L. (2002). SPEA2: Improving the strength Pareto evolutionary algorithm for multiobjective optimization. In Proceedings of the Evolutionary Methods for Design, Optimisation and Control with Application to Industrial Problems Conference, (EUROGEN 2001), pp. 95-100.

\section{Appendix A Dominance-based Edges from Rotations}

Here we present an alternative method for identifying the edges of mutually nondominating sets. The motivation for this method comes from the fact that, as illustrated in Figure 15(a), the non-dominated elements of a set define a portion of the set's boundary. The edges of the set might therefore be identified as the union of the non-dominated points as the set is rotated.

In general, the points of a multi-criterion non-dominated set are not coplanar, so in order to use this idea with many-criterion non-dominated sets, we first project the points in $\mathcal{Y}$ onto a plane in which they remain mutually non-dominating.

Without loss of generality, we assume that the elements of $\mathcal{Y}$ are non-negative $\left(y_{n m} \geq 0\right.$ for all $\left.n, m\right)$. Then the simplex defined by the numbers $\left\{\lambda_{m}: \lambda_{m}>0\right\}_{m=1}^{M}$ is the portion of the (hyper-) plane which lies in the positive orthant and which intersects the coordinate axes at distances $\lambda_{m}$ from the origin, as illustrated in Figure 15(b). The simplex is therefore the segment of the plane in the positive orthant defined by

$$
\mathbf{n} \cdot \mathbf{y}=d \quad y_{m} \geq 0, \quad m=1, \ldots, M
$$

where the elements of the unit vector $\mathbf{n}$ normal to the simplex are $n_{m}=d / \lambda_{m}$ and the perpendicular distance to the origin $d$ can be found as

$$
d^{-2}=\sum_{m=1}^{M} \lambda_{m}^{-2}
$$

Points are then projected onto the simplex by:

$$
\hat{\mathbf{y}}_{n}=\mathbf{y}_{n} /\left(\mathbf{y}_{n} \cdot \mathbf{n}\right),
$$




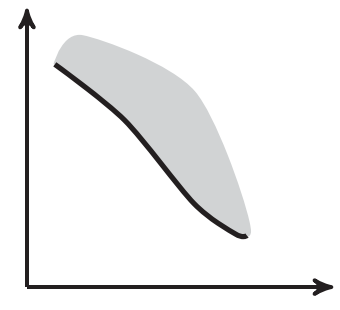

(a)

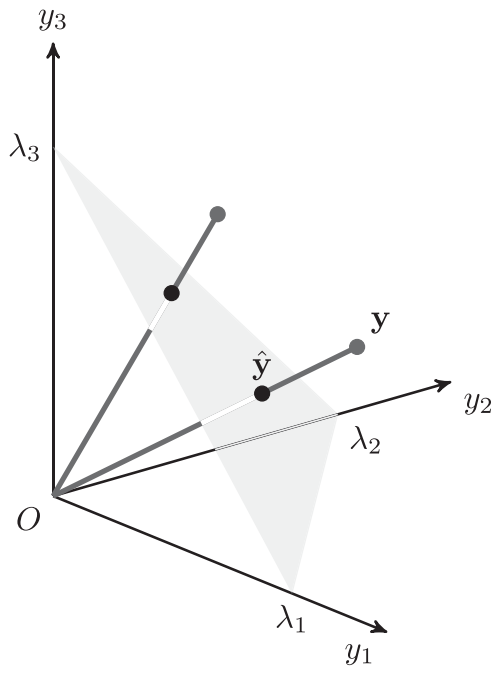

(b)

Figure 15: (a) Non-dominated points identify the boundary of a set. Non-dominated regions of the set in the plane are marked in black. (b) Projection of a point $\mathbf{y}$ onto the simplex defined by $\left\{\lambda_{1}, \lambda_{2}, \lambda_{3}\right\} ; \mathbf{y}$ is projected to $\hat{\mathbf{y}}$.

and we emphasise that the projection of $\mathcal{Y}$ results in a set of mutually non-dominating points. The choice of the $\lambda_{m}$ clearly affects the particular projection, but since the dominance relations between the points are unaffected, the method is not sensitive to the precise values. We have used $\lambda_{m}=$ median $_{\mathbf{y} \in \mathcal{Y}} y_{m}$ here.

To identify the edges of this set, we now rotate the $\hat{\mathbf{y}}_{n}$ in the plane of the simplex and identify the non-dominated points in each rotation.

Coordinates for the $\hat{\mathbf{y}}_{n}$ on the simplex may be simply found by projecting the $\hat{\mathbf{y}}_{n}$ onto their principal components or equivalently by singular value decomposition. Let $\hat{\mathbf{Y}}=\left[\hat{\mathbf{y}}_{1}-\mu, \ldots, \hat{\mathbf{y}}_{N}-\mu\right]$ be the matrix whose columns are the mean-centred $\hat{\mathbf{y}}_{n}$ and let $\hat{\mathbf{Y}}=\mathbf{U} \boldsymbol{\Sigma} \mathbf{V}^{T}$ be the singular value decomposition of $\hat{\mathbf{Y}}$. Then the first $M-1$ columns of the orthonormal matrix $\mathbf{U}$ span the subspace of the simplex (the last column is a vector normal to the simplex). Coordinates of $\mathbf{y}_{n}$ mapped to the simplex are thus

$$
\tilde{\mathbf{y}}_{n}=U_{M-1}^{T}\left(\hat{\mathbf{y}}_{n}-\mu\right)
$$

where $U_{M-1}$ denotes the matrix of the first $M-1$ columns of $U$.

Rather than exhaustively quartering all rotations, we generate rotations $\mathbf{Q}$ at random. Uniformly distributed random rotations are generated by, for example, a $Q R$ decomposition of $(M-1) \times(M-1)$ dimensional matrices whose elements are Gaussian distributed. The signs of the columns of $\mathbf{Q}$ are arbitrary, so in all $2^{M-1}$ rotations can be cheaply generated from a single QR decomposition by appropriately flipping the signs of the columns of $\mathbf{Q}$.

As each rotation is generated the non-dominated points nondom $\left(\left\{\mathbf{Q} \tilde{\mathbf{y}}_{n} \mid \mathbf{y}_{n} \in \mathcal{Y}\right\}\right)$ are found and the corresponding $\mathbf{y}$ added to the identified edge points.

This method of determining edges is demonstrated for the hourglass set in Figure 16. It is clear that a reasonable set of points has been identified as edge points, both on the upper, concave portion and on the lower, convex section. However, the relationship of these edge points to those found with the attainment surface or by projection onto 


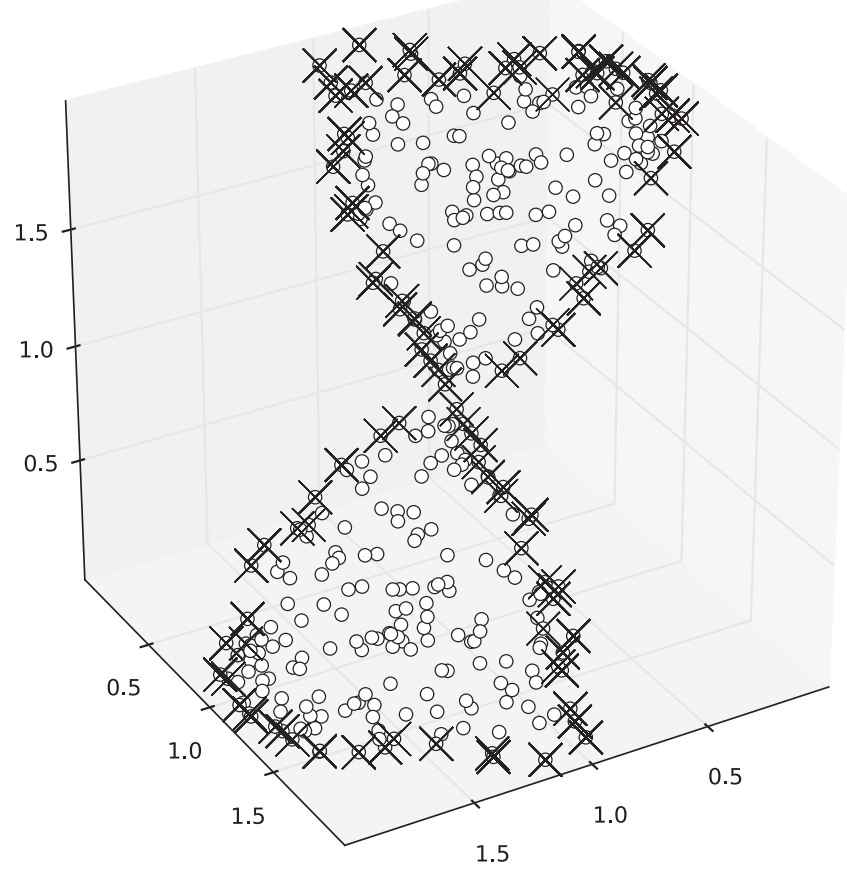

Figure 16: Edge points of the hourglass set identified from non-dominated points after projection onto the simplex and 64 random rotations and associated flips.

subsets of the criteria is not clear. We note also that if $\mathcal{Y}$, when projected onto the simplex, has "deep and narrow" concavities, then this method will be unable to locate edge points in the concavities.

We also applied this method to the task of identifying the edge of the nine-criterion radar data. However, like the other methods, it too identifies all points as being on the edge. Again, this is due to the relative lack of discrimination provided by the dominance relation in high dimensional spaces. 
